\title{
¿CONDICIONAN EL GÉNERO Y LA EDAD EL NIVEL DE COMPETENCIA DIGITAL? UN ESTUDIO CON ESTUDIANTES UNIVERSITARIOS
}

\section{Do Gender and Age Affect the Level of Digital Competence? A Study with University Students}

\author{
Dr. Marcos CABEZAS GONZÁLEZ \\ Profesor Contratado Doctor, Universidad de Salamanca, España, mcabezasgo@usal.es \\ (D) orcid.org/0000-0002-3743-5839
}

Dra. Sonia CASILLAS MARTÍN

Profesora Contratada Doctora, Universidad de Salamanca, España, scasillasma@usal.es

(iD) orcid.org/0000-0001-5304-534X

\section{Dra. Manuela SANCHES-FERREIRA}

Profesora Coordinadora en la Unidad de Educación Especial de Ciencia y Técnica de Inclusión, Universidad de Oporto, Portugal, manuelaferreira@ese.ipp.pt

(D) orcid.org/0000-0002-4693-3928

\section{Dr. Fernando Luís TEIXEIRA DIOGO}

Profesor Adjunto en la Unidad Técnico Científica de Ciencias de la Educación, Universidad de Oporto, Portugal, fdiogo@ese.ipp.pt

Fecha de recepción del artículo: 04/10/2017

Fecha de aceptación definitiva: 12/10/2017

\begin{abstract}
RESUMEN
Las características de las Tecnologías de la Información y la Comunicación (TIC) y su implantación a nivel global han originado cambios significativos en diferentes ámbitos, de manera especial en los institucionales. En este artículo se presentan los resultados de una investigación cuyo propósito es el de conocer el nivel de competencia digital de estudiantes universitarios de titulaciones de educación y verificar si las variables de género y edad tienen alguna influencia en el mismo. Se empleó una metodología cuantitativa, con un método no experimental, descriptivo e inferencial, y el cuestionario digital como instrumento para la recogida de información sobre las dimensiones de conocimiento, manejo y actitudes hacia las TIC. Los datos fueron analizados a partir de una comparación de medias, utilizando pruebas no paramétricas. Los resultados manifiestan que la muestra estudiada se autoevalúa de manera negativa en el conocimiento de conceptos TIC, positivamente en el manejo de dispositivos, herramientas y servicios, mostrando una actitud muy positiva hacia la tecnología. Respecto a las variables estudiadas, existen diferencias significativas en el conocimiento y el manejo, a favor de los hombres y respecto a la actitud, a favor de los sujetos de mayor edad.
\end{abstract}

Palabras clave: Competencia digital; Tecnologías de la Información y la Comunicación; formación inicial; género; edad. 


\begin{abstract}
The characteristics of Information and Communication Technologies (ICT) and their implementation at the global level have led to significant changes in different areas, especially institutional ones. This article presents the results of a research study whose purpose was to learn the level of digital competence of university students of education and to verify whether the variables of gender and age have any influence on it. A quantitative methodology was used, with a non-experimental, descriptive and inferential method, and the digital questionnaire was employed as an instrument for collecting information on the dimensions of knowledge and management of ICTs as well as attitudes towards them. The data were analysed based on a comparison of means, using non-parametric tests. The results show that the sample studied self-evaluated negatively their knowledge of ICT concepts, considered positive their management of devices, tools and services, and showed a very positive attitude toward technology. Regarding the variables studied, significant differences were found in favour of men in relation to knowledge and management, and in favour of older subjects with regard to attitude.
\end{abstract}

Key words: Digital competence; Information and Communication Technologies; initial training; gender; age.

\title{
1. INTRODUCCIÓN
}

En 1997, Castells escribió: «por primera vez en la historia, la mente humana es una fuerza productiva directa, no solo un elemento decisivo del sistema de producción» (p. 62). En la actualidad esta afirmación sigue teniendo plena vigencia y se contextualiza en un tipo de sociedad en la que los cambios económicos y sociales han transformado la base material de la sociedad, y el procesamiento y la transferencia de información se han convertido en los pilares fundamentales de la productividad y el poder, y en donde la base de los procesos productivos se sustenta en el conocimiento de las personas (Alonso, 2013, Castells, 2006).

En esta Sociedad denominada de la Información y el Conocimiento, caracterizada por su complejidad y su creciente globalización, las Tecnologías de la Información y la Comunicación (TIC) han originado profundos cambios en la construcción del conocimiento y en el aprendizaje (Guillén, 2014) que ponen de manifiesto la necesidad de una formación diferente a la de la sociedad industrial y postindustrial (Cabero, 2014; Gisbert, Spuny y González, 2011), que desarrolle nuevos conocimientos, procedimientos y actitudes, y en la que la competencia digital sea un elemento esencial en la alfabetización académica (Roldán y Zabaleta, 2016).

Las características de las TIC y su implantación a nivel global han originado cambios significativos en diferentes ámbitos, de manera especial en los institucionales. La posibilidad de acumular información, su rápida transmisión, la desaparición de las barreras espaciales y temporales, el empleo de múltiples medios y soportes son, entre otras, algunas de las características que explican el gran potencial de cambio que aportan estas tecnologías.

En este contexto, parece necesario que, entre las capacidades fundamentales que los universitarios deberían desarrollar, se encuentren: (a) competencia para entender conceptos y problemas complejos; (b) alfabetización digital; (c) uso avanzado de las TIC; (d) habilidades sociales y comunicativas para el desarrollo personal, social y laboral; (e) trabajo colaborativo (Dumont, Istance y Benavides, 2010).

La Unión Europea (2007) define ocho competencias fundamentales para el aprendizaje permanente, entre las que encontramos la competencia digital, entendida como:

conjunto de conocimientos, habilidades, actitudes, estrategias y conciencia que se requieren cuando se utilizan las TIC y los medios digitales para realizar tareas; resolver problemas; comunicar; gestionar la información; colaborar; crear y compartir contenido; y construir conocimiento de manera efectiva, eficiente, apropiada, crítica, creativa, autónoma, flexible, ética, reflexiva para el trabajo, el ocio, la participación, el aprendizaje y la socialización (Ferrari, 2012, p. 30). 
En los últimos años, son diferentes las investigaciones que han estudiado la competencia digital autoevaluada, en el campo educativo, en diferentes contextos y niveles (Cabezas, Casillas y Pinto, 2014; Edelhard, 2015; Fernández, Román y El Homrani, 2017; Fernández y Torres, 2015; Maderick, Shaoan, Hartley y Marchand, 2015; Mork y Krumsvik, 2016; Rambousek, Stipek \& Vanková, 2016). También las que se han centrado en la influencia entre diferentes factores y la competencia digital (González, Galvis y Sánchez, 2015; Nasah, DaCosta, Kinsell y Seok, 2010; Ricoy, Feliz y Sevillano, 2010). Recogiendo esta doble línea de trabajo, este artículo presenta los resultados de un estudio realizado con el propósito de conocer el nivel de competencia digital de estudiantes universitarios de titulaciones de educación y verificar si las variables de género y edad tienen alguna influencia en el mismo.

Es necesario que la competencia digital sea una capacidad transversal en la formación inicial de los profesionales de la Educación, porque estos deberán desarrollar su quehacer profesional en un mundo complejo e interconectado enfrentado constantemente a cambios acelerados de tipo tecnológico, cultural, económico, informativo y demográfico (Kampylis, Punie y Devine, 2015).

\section{OBJETIVOS}

El principal objetivo que se pretende conseguir es el de conocer el nivel de competencia digital manifestado por los estudiantes universitarios de diferentes titulaciones de grado y postgrado de la Facultad de Psicología y Ciencias de la Educación de la Universidad de Oporto (Portugal).

De manera operativa, pretendemos averiguar si los conocimientos, el manejo y las actitudes hacia las TIC autopercibidos, son diferentes en función de las variables de control género y edad.

\section{Metodología utilizada}

Se empleó una metodología cuantitativa, con un método no experimental, descriptivo e inferencial (Arnal, del Rincón y Latorre, 1992; Kerlinger y Lee, 2002), y la recogida de información se realizó mediante un cuestionario en soporte electrónico (Berends, 2006; Kerlinger y Lee, 2002). Las dimensiones estudiadas se presentan en la figura 1.

Fig. 1. Modelo de investigación

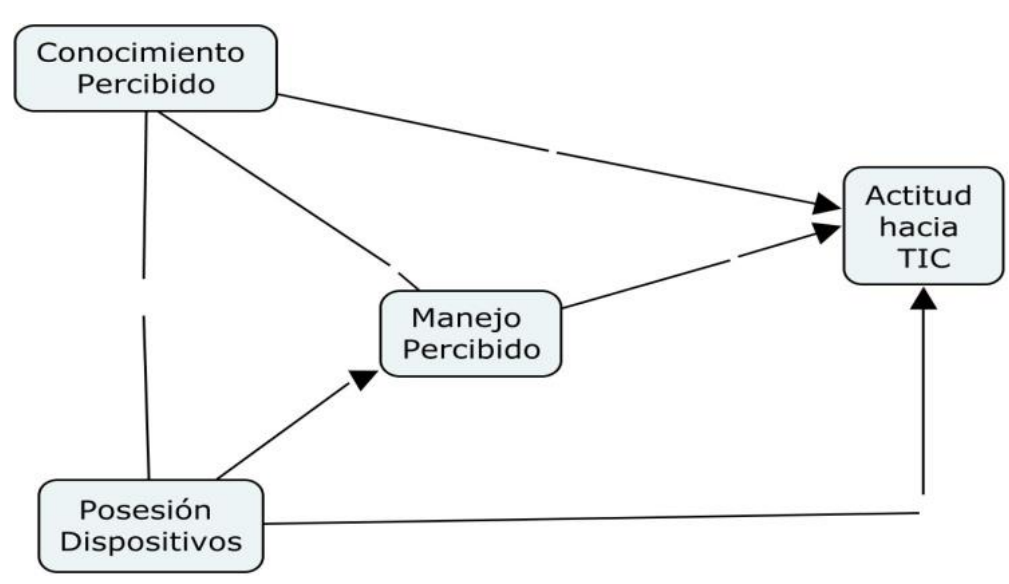

Fuente: elaboración propia. 


\subsection{MUESTRA}

La recogida de datos estuvo dirigida a todos los estudiantes de Educación que cursaron algún Grado o Postgrado en la Facultad de Psicología y Ciencias de la Educación de la Universidad de Oporto (Portugal). Siguiendo un tipo de muestreo aleatorio simple (Fraenkel, Wallen y Hyun, 2012), la muestra final quedó compuesta por un total de 70 estudiantes (tabla 1), de los cuales, el 13,4\% son hombres y el 86,6\% mujeres, con edades comprendidas entre los 18 y los 57 años. El 22,4\% procede del contexto rural y el $77,6 \%$ del contexto urbano.

Tabla 1. Titulaciones de los alumnos

\begin{tabular}{|l|}
\hline \multicolumn{1}{|c|}{ TITULACIONES QUE PARTICIPAN EN LA MUESTRA } \\
\hline \multicolumn{1}{|c|}{ Grado } \\
\hline Equivalência à licenciatura \\
\hline Licenciatura contabilidade e gestao \\
\hline licenciatura de Tradução e Interpretação em Língua Gestual Portuguesa \\
\hline Licenciatura Educação Básica \\
\hline Licenciatura em Matemática (Ensino de) \\
\hline Licenciatura em terapêutica da fala \\
\hline \multicolumn{1}{|c|}{ Máster } \\
\hline Mestrado Educação Especial: Multideficiência e Problemas de Cognição \\
\hline Mestrado Educação Pré-Escolar \\
\hline Mestrado Educação Pré-Escolar e Ensino do ${ }^{\circ}$ Ciclo do Ensino Básico \\
\hline Mestrado em Ensino do $1^{\circ}$ e $2^{\circ}$ ciclo do Ensino Básico \\
\hline Mestrado Ensino do $1 .^{\circ}$ Ciclo do Ensino Básico e de Matemática e Ciências Naturais no 2. ${ }^{\circ}$ Ciclo do Ensino Básico \\
\hline $\begin{array}{l}\text { Mestrado Estudos Profissionais Especializados em Educação - Especialização em Administração das Organizações } \\
\text { Educativas }\end{array}$ \\
\hline
\end{tabular}

Fuente: elaboración propia.

De los futuros profesionales de la educación que componen la muestra, un 98,5\% disponen de ordenador portátil, el 82,1\% disponen de un Smartphone, un 59,7\% tienen ordenador de sobremesa, el $74,6 \%$ cámara de fotos digital para uso personal, y un 59,7\% y 56,7\% disponen de Tablet y Sistema de Posicionamiento Global (GPS) respectivamente. Son menos los que tienen cámara de video digital $(35,8 \%)$ y prácticamente ninguno dispone de un dispositivo para la lectura de libros electrónicos $(9 \%)$, y solo un $6 \%$ poseen algún tipo de wearable o dispositivo electrónico que se incorpora en alguna parte del cuerpo interactuando continuamente con el usuario y con otros dispositivos con la finalidad de realizar alguna función específica.

\subsection{INSTRUMENTO DE RECOGIDA DE INFORMACIÓN}

Se empleó un cuestionario de respuesta directa, pre-codificado y transversal, diseñado ad hoc para el análisis de conocimientos, manejo y actitudes sobre las TIC que poseen los alumnos universitarios.

Dicho instrumento consta de 91 ítems, tres de los cuales son variables de identificación. Se utilizaron dos escalas, una cerrada dicotómica con opciones de respuesta de sí o no y otra con valoración tipo Likert de 1 a 10 con la intención de utilizar una manera de valorar similar a la evaluación académica (con las que los estudiantes están familiarizados). Para el bloque sobre manejo de dispositivos la escala se interpreta con 1 muy incompetente y 10 muy competente; en el bloque relativo a la actitud se interpreta como 1 muy en desacuerdo y 10 muy de acuerdo. El cuestionario inicial fue mejorado tras la aplicación de una prueba piloto y sometido a juicio de expertos para su validación. Para asegurar la cumplimentación correcta e independiente por parte de los estudiantes fue aplicado directamente por 
los investigadores. La fiabilidad de la escala, medida a través del estadístico $\alpha$ de Cronbach, resulta muy satisfactoria $(\alpha=.963)$.

Todas las competencias estudiadas a partir de los diferentes ítems del cuestionario se estructuran en cinco bloques generales:

Bloque 1. Identificación (ID). Datos de identificación: género, edad, lugar de origen, estudios del padre y de la madre, nota de acceso a la Universidad, titulación y curso.

Bloque 2. Posesión (PS). Dispositivos TIC que poseen.

Bloque 3. Conocimiento (CN). Autoevaluación de conocimientos sobre conceptos relacionados con las TIC (CN_01) y de conocimientos de dispositivos TIC (CN_02).

Bloque 4. Manejo (MJ). Autoevaluación de capacidades para manejar dispositivos TIC (MJ_01), herramientas TIC (MJ_02) y servicios TIC (MJ_03).

Bloque 5. Actitud (AC). Valoración de las TIC de acuerdo a su necesidad e importancia para el futuro profesional de la educación.

\subsection{ANÁLISIS DE LOS DATOS}

El tratamiento estadístico se realizó por medio del programa Statistical Package for the Social Sciences (SPSS v.22) y los datos fueron analizados de manera descriptiva por medio del cálculo de estadísticos descriptivos básicos, y de manera inferencial a partir de una comparación de medias utilizando las pruebas no paramétricas de U Mann- Whitney para la variable dicotómica género y la de Kruskal-Wallis para la variable edad.

Al comprobarse la no existencia de normalidad en los resultados mediante la prueba de Kolmogorov-Smirnov y de homocedasticidad (prueba de Levene) y teniendo en cuenta el tamaño de la muestra, se optó por la utilización de estas pruebas.

\section{Resultados}

Este apartado se estructura en dos subapartados. Por un lado, presentamos los resultados obtenidos en el análisis descriptivo de los datos, y por el otro, los obtenidos con el análisis inferencial. Estos resultados se centran en los bloques tres, cuatro y cinco (CN, MJ y AC).

\subsection{ANÁLISIS DESCRIPTIVO}

\subsubsection{CONOCIMIENTOS SOBRE CONCEPTOS Y DISPOSITIVOS TIC (CN)}

En la tabla 2 se recogen los estadísticos descriptivos básicos de la autoevaluación que realizan los estudiantes sobre su conocimiento de conceptos y dispositivos TIC. 
Tabla 2. Estadísticos descriptivos sobre conocimientos TIC

\begin{tabular}{|c|c|c|c|c|}
\hline \multicolumn{5}{|c|}{ CONOCIMIENTOS (CN) } \\
\hline Conceptos (CN_01) & $\overline{\mathbf{x}}$ & SD & Mínimo & Máximo \\
\hline $\begin{array}{l}\text { Sei o que são as TIC (Tecnologias da Informação e da Comu- } \\
\text { nicação) }\end{array}$ & 8,82 & 1,585 & 4 & 10 \\
\hline $\begin{array}{l}\text { Sei o que são as TAC (Tecnologias da Aprendizagem e do } \\
\text { Conhecimento) }\end{array}$ & 4,42 & 2,960 & 0 & 10 \\
\hline $\begin{array}{l}\text { Sei o que são as TEP (Tecnologias para o Empoderamento e a } \\
\text { Participação) }\end{array}$ & 2,34 & 2,626 & 0 & 10 \\
\hline Sei o que é a Web 2.0 & 3,76 & 3,504 & 0 & 10 \\
\hline Sei o que é Web 3.0 & 3,75 & 3,560 & 0 & 10 \\
\hline Sei o que é Wikipedia & 9,49 & 1,006 & 6 & 10 \\
\hline Sei o que é a blogosfera & 6,28 & 3,700 & 0 & 10 \\
\hline Sei o que são é um podcast & 4,99 & 3,776 & 0 & 10 \\
\hline Sei o que é um marcador social & 3,64 & 3,467 & 0 & 10 \\
\hline Sei o que é a sindicação ou a redifução web & 1,90 & 2,394 & 0 & 8 \\
\hline Sei o que é um mashup & 2,57 & 3,281 & 0 & 10 \\
\hline Sei o que é um objecto de aprendizagem & 6,43 & 3,201 & 0 & 10 \\
\hline Sei o que é o E-learning & 7,69 & 3,056 & 0 & 10 \\
\hline Sei o que é o M-learning & 3,51 & 3,665 & 0 & 10 \\
\hline Sei o que é o B-learning & 5,30 & 4,000 & 0 & 10 \\
\hline Sei o que é um MOOC & 1,40 & 2,431 & 0 & 10 \\
\hline Sei o que é o armazenamento numa nuvem & 7,42 & 3,702 & 0 & 10 \\
\hline Sei o que é a realidade virtual (RV) & 6,42 & 3,363 & 0 & 10 \\
\hline Sei o que é a realidade aumentada (RA) & 4,12 & 3,629 & 0 & 10 \\
\hline Sei o que é o copyleft & 1,76 & 2,877 & 0 & 10 \\
\hline Sei o que é uma licença Creative Commons & 1,51 & 2,464 & 0 & 10 \\
\hline Sei o que é a brecha digital & 1,64 & 2,353 & 0 & 10 \\
\hline Sei o que é a E-exclusão & 3,60 & 3,774 & 0 & 10 \\
\hline Sei o que é a E-inclusão & 3,72 & 3,741 & 0 & 10 \\
\hline Sei o que é a alfabetização digital & 4,84 & 3,616 & 0 & 10 \\
\hline Sei o que é o ciberativismo & 4,16 & 3,383 & 0 & 10 \\
\hline Sei o que é a E-participação & 3,37 & 3,274 & 0 & 10 \\
\hline Sei o que é o empoderamento & 2,76 & 3,238 & 0 & 10 \\
\hline Dispositivos TIC (CN_02) & $\overline{\mathbf{x}}$ & SD & Mínimo & Máximo \\
\hline Sei o que é uma tablet & 9,52 & 1,133 & 4 & 10 \\
\hline Sei o que é um Smartphone & 9,63 &, 850 & 6 & 10 \\
\hline Sei o que é um e-Book & 8,48 & 2,727 & 0 & 10 \\
\hline $\begin{array}{l}\text { Sei o que é uma PDI (Lousa Digital Interactiva/Quadro Bran- } \\
\text { co Interativo) }\end{array}$ & 7,78 & 3,316 & 0 & 10 \\
\hline Sei o que é um GPS & 9,40 & 1,244 & 5 & 10 \\
\hline Sei o que é um wearable (que se pode levar ou mesmo vestir) & 3,81 & 3,967 & 0 & 10 \\
\hline
\end{tabular}

Fuente: elaboración propia. 
Los estudiantes no conocen la mayoría de conceptos relacionados con la tecnología (CN_01). Se autoevalúan con sobresaliente cuando se les pregunta por el concepto de wikipedia $(9,49)$, con notable en el concepto sobre qué son las TIC $(8,82)$, en qué es e-learnig $(7,69)$ y en el concepto de almacenamiento en la nube $(7,42)$. En menor medida, con puntuaciones cercanas al aprobado, valoran su conocimiento de conceptos como la blogosfera, realidad virtual y b-learning. En el resto de términos se otorgan una puntuación de suspenso.

Cuando se les pregunta por el conocimiento de dispositivos (CN_02), las valoraciones son muy superiores. Se autoevalúan con una puntuación muy alta (notable-sobresaliente) su conocimiento sobre lo que es un Smartphone, un eBook, un GPS, una Tablet y una Pizarra Digital Interactiva (PDI), pero reconocen no saber lo que es un wearable.

\subsubsection{MANEJO DE DISPOSITIVOS, HERRAMIENTAS Y SERVICIOS TIC (MJ)}

Respecto al manejo de dispositivos (MJ_01), se evalúan con puntuaciones altas (tabla 3) entre notable-sobresaliente, y consideran que disponen de una mayor capacidad para el manejo de ordenadores y Tablet, y una menor competencia para el manejo de lector de eBook. Se suspenden, con una puntuación muy baja, en el manejo de wearables.

Tabla 3. Estadísticos descriptivos sobre manejo de dispositivos TIC

\begin{tabular}{lcccc}
\hline & MANEJO (MJ) & & & \\
\hline Dispositivos (MJ_01) & $\overline{\mathbf{x}}$ & SD & Mínimo & Máximo \\
\hline Sei usar computadores & 8,88 & 1,581 & 4 & 10 \\
\hline Sei usar uma câmara digital de fotos & 8,66 & 1,771 & 2 & 10 \\
\hline Sei usar uma câmara vídeodigital & 7,90 & 2,541 & 0 & 10 \\
\hline Sei usar smartphones & 8,58 & 2,203 & 1 & 10 \\
\hline Sei usar tablets & 8,82 & 1,833 & 2 & 10 \\
\hline Sei usar um leitor de eBooks & 6,21 & 3,391 & 0 & 10 \\
\hline Sei usar um GPS & 7,87 & 2,552 & 0 & 10 \\
\hline Sei usar wearables & 2,45 & 3,032 & 0 & 10 \\
\hline
\end{tabular}

Si tenemos en cuenta las herramientas (MJ_02), las puntuaciones siguen siendo altas (tabla 4). Consideran que tienen capacidades para el manejo de las herramientas TIC planteadas, sobre todo para usar redes sociales, herramientas de comunicación (WhatsApp, correo electrónico, videoconferencia, chat, foros, etc.) y herramientas de edición de texto. Sus peores valoraciones se refieren al manejo de aquellas que sirven para la edición de imagen y sonido. Si nos detenemos en las desviaciones típicas, comprobamos que en la mayoría de los aspectos son elevadas, lo que nos indica que no hay acuerdo en las respuestas otorgadas. 
Tabla 4. Estadísticos descriptivos sobre manejo de herramientas TIC

\begin{tabular}{|c|c|c|c|c|}
\hline \multicolumn{5}{|c|}{ MANEJO (MJ) } \\
\hline Herramientas (MJ_02) & $\overline{\mathbf{x}}$ & SD & Mínimo & Máximo \\
\hline $\begin{array}{l}\text { Sei usar ferramentas informáticas de escritório (processado- } \\
\text { res de textos, apresentações, bases de dados, folhas de cálcu- } \\
\text { lo...) }\end{array}$ & 7,96 & 2,041 & 2 & 10 \\
\hline Sei usar ferramentas de edição de imagem, som, vídeo... & 6,85 & 2,548 & 0 & 10 \\
\hline $\begin{array}{l}\text { Sei usar ferramentas de procura e documentação (buscado- } \\
\text { res, publicações eletrônicas, recursos didáticos, objectos de } \\
\text { aprendizagem...) }\end{array}$ & 7,72 & 2,282 & 0 & 10 \\
\hline $\begin{array}{l}\text { Sei usar ferramentas de trabalho em colaboração (blogs, } \\
\text { wikis, webquest, google drive, outlook...) }\end{array}$ & 7,39 & 2,283 & 1 & 10 \\
\hline $\begin{array}{l}\text { Sei usar ferramentas de organização do tempo (calendário o } \\
\text { agenda eletrônica, programação de reuniões, programação de } \\
\text { eventos...) }\end{array}$ & 7,28 & 2,610 & 0 & 10 \\
\hline $\begin{array}{l}\text { Sei usar ferramentas de comunicação (whatsApp, correio } \\
\text { eletrónico, videoconferência, chat, foros...) }\end{array}$ & 8,12 & 2,273 & 0 & 10 \\
\hline $\begin{array}{l}\text { Sei usar ferramentas para a formação (plataformas e-learning, } \\
\text { moodle...) }\end{array}$ & 7,48 & 2,414 & 0 & 10 \\
\hline $\begin{array}{l}\text { Sei usar ferramentas de armazenamento na nuvem (Dro- } \\
\text { pbox, OneDrive, GoogleDrive, Box...) }\end{array}$ & 7,54 & 2,721 & 0 & 10 \\
\hline $\begin{array}{l}\text { Sei usar redes sociais (faceBook, twitter, linkedin, instagram, } \\
\text { youtube...) }\end{array}$ & 8,70 & 1,784 & 1 & 10 \\
\hline
\end{tabular}

Fuente: elaboración propia.

Es en la autoevaluación del uso de servicios (MJ_03) en donde las valoraciones son muy dispersas (tabla 5), existiendo mucha diferencia entre unos alumnos y otros. Sorprende, por su escasa utilización, la valoración que otorgan respecto al manejo de la app de mensajería instantánea denominada Wasap. Reconocen utilizar bastante la red social de FaceBook, pero no la de Twitter. Valoran, con una nota próxima al notable, su manejo de Internet para publicar y compartir fotos y videos; en menor medida, para compartir y publicar presentaciones. Se suspenden a la hora de compartir y publicar archivos de audio, y en consultar y publicar en wikis. Reconocen no tener blog, ni sitio web propio, ni tampoco manejan el almacenamiento en la nube. 
Tabla 5. Estadísticos descriptivos sobre manejo de servicios TIC

\begin{tabular}{|c|c|c|c|c|}
\hline \multicolumn{5}{|c|}{ MANEJO (MJ) } \\
\hline Servicios (MJ_03) & $\overline{\mathbf{x}}$ & SD & Mínimo & Máximo \\
\hline Utilizo a rede social Twitter & ,91 & 2,353 & 0 & 10 \\
\hline Utilizo a rede social FaceBook & 8,19 & 2,955 & 0 & 10 \\
\hline Utilizo WhatsApp & 5,60 & 4,053 & 0 & 10 \\
\hline $\begin{array}{l}\text { Utilizo serviços da Internet para publicar e partilhar } \\
\text { fotografias digitais }\end{array}$ & 6,63 & 3,554 & 0 & 10 \\
\hline $\begin{array}{l}\text { Utilizo serviços da Internet para publicar e partilhar } \\
\text { vídeos }\end{array}$ & 5,70 & 3,750 & 0 & 10 \\
\hline $\begin{array}{l}\text { Utilizo serviços da Internet para publicar e partilhar } \\
\text { apresentações }\end{array}$ & 5,16 & 3,768 & 0 & 10 \\
\hline $\begin{array}{l}\text { Utilizo serviços da Internet para publicar e partilhar } \\
\text { arquivos de audio }\end{array}$ & 4,07 & 3,661 & 0 & 10 \\
\hline $\begin{array}{l}\text { Utilizo serviços de chat, comunicação, videoconferência } \\
\text { (Skype, Gooogle Hangouts...) }\end{array}$ & 6,33 & 3,431 & 0 & 10 \\
\hline Tenho o meu próprio blog & 1,12 & 2,821 & 0 & 10 \\
\hline Tenho o meu próprio sitio web &, 75 & 2,312 & 0 & 10 \\
\hline Consulto wikis & 2,70 & 3,402 & 0 & 10 \\
\hline Publico conteúdos nas wikis &, 57 & 1,777 & 0 & 10 \\
\hline $\begin{array}{l}\text { Utilizo serviços da Internet para agregar e alojar con- } \\
\text { teúdos (Diigo, Mr. Wong, Delicious) }\end{array}$ &, 54 & 1,761 & 0 & 10 \\
\hline $\begin{array}{l}\text { Utilizo a nuvem para alojar e partilhar arquivos (Google } \\
\text { Drive, One Drive, Dropbox, Box...) }\end{array}$ & 6,28 & 3,334 & 0 & 10 \\
\hline
\end{tabular}

Fuente: elaboración propia.

\subsubsection{ACTITUD HACIA LAS TIC}

Es muy positiva en todas sus dimensiones (tabla 6). Si tenemos en cuenta las medias obtenidas en cada uno de los ítems relativos a esta variable, consideran que las TIC son necesarias y útiles para su futuro profesional $(8,43)$ y que les ayudarán a economizar esfuerzo, tanto en su trabajo académico en la Universidad como en el profesional $(8,07$ y 7,87). Del mismo modo, piensan que el uso de las mismas puede ayudarles en su desarrollo profesional, además de ser un medio útil para seguir formándose $(8,48$ y 8,09$)$. En menor medida, manifiestan que las TIC son un medio frío y distante $(5,75)$. 
Tabla 6. Estadísticos descriptivos sobre Actitud hacia las TIC

\begin{tabular}{|c|c|c|c|c|}
\hline \multicolumn{5}{|c|}{ ACTITUD TIC (AC) } \\
\hline & $\overline{\mathbf{x}}$ & SD & Mínimo & Máximo \\
\hline $\begin{array}{l}\text { As TIC são um meio de comunicação frio e distante. Não há nada } \\
\text { como o face a face }\end{array}$ & 5,75 & 2,566 & 0 & 10 \\
\hline $\begin{array}{l}\text { As TIC são necessárias e úteis para a minha futura profissão de } \\
\text { educador }\end{array}$ & 8,43 & 1,734 & 3 & 10 \\
\hline $\begin{array}{l}\text { As TIC ajudam-me a economizar o esforço e o trabalho académico } \\
\text { durante o meus estudos }\end{array}$ & 8,07 & 2,055 & 2 & 10 \\
\hline $\begin{array}{l}\text { As TIC ajudar-me-ão a economizar o esforço e o trabalho na min- } \\
\text { ha futura vida profissional como Educador }\end{array}$ & 7,87 & 2,138 & 2 & 10 \\
\hline $\begin{array}{l}\text { As TIC são um meio útil que me ajudarão a continuar a minha } \\
\text { formação como futuro profissional da Educação }\end{array}$ & 8,48 & 1,778 & 3 & 10 \\
\hline $\begin{array}{l}\text { É necessário que as TIC se convertam em ferramentas habituais no } \\
\text { desenvolvimento profissional do Educador }\end{array}$ & 8,09 & 2,158 & 3 & 10 \\
\hline $\begin{array}{l}\text { O Educador tem de ser um profissional competente para utilizar as } \\
\text { TIC na sua profissão }\end{array}$ & 7,96 & 1,829 & 3 & 10 \\
\hline $\begin{array}{l}\text { Sinto-me preparado para integrar as TIC no meu futuro profissio- } \\
\text { nal como Educador }\end{array}$ & 8,16 & 2,086 & 2 & 10 \\
\hline
\end{tabular}

Fuente: elaboración propia.

\subsection{ANÁLISIS INFERENCIAL}

A continuación, se exponen los resultados comparando las medias en función de las variables género y edad, presentando las diferencias significativas encontradas a partir del análisis inferencial realizado.

\subsubsection{VARIABLE DIFERENCIADORA GÉNERO}

Los hombres se autoevalúan por encima de las mujeres en el bloque de conocimiento $(\mathrm{CN})$ (gráfico 1), valorando mejor su saber en conceptos y dispositivos TIC, frente a las mujeres, cuyo conocimiento no llega a una puntuación de cinco.

Gráfico 1. Medias del bloque CN en función del género

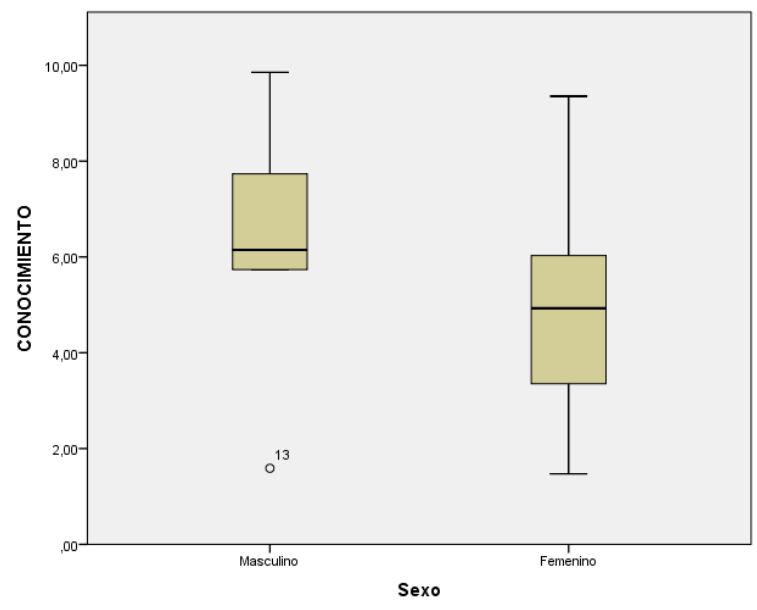

Fuente: elaboración propia 
Respecto al manejo (MJ), vuelven a ser los hombres los que se perciben mejor en este bloque (gráfico 2), tanto en la utilización de dispositivos, como de herramientas y de servicios. Las futuras educadoras, creen manejar peor la tecnología, otorgándose una media de aprobado.

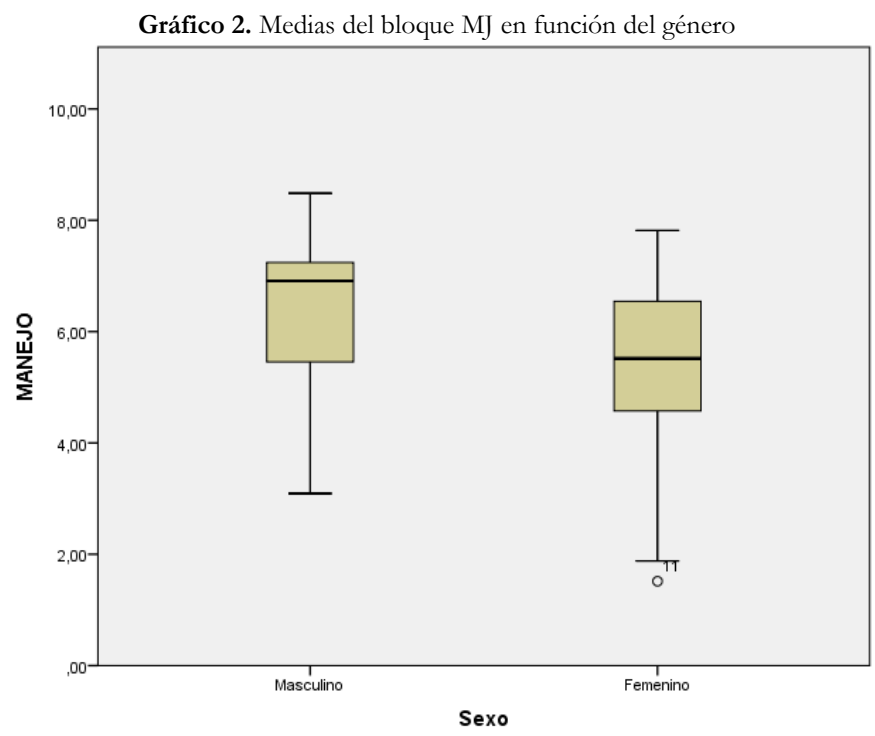

Fuente: elaboración propia.

Y también son ellos los que manifiestan una mejor actitud hacia las TIC (AC) (gráfico 3), siendo en este caso menores las diferencias.

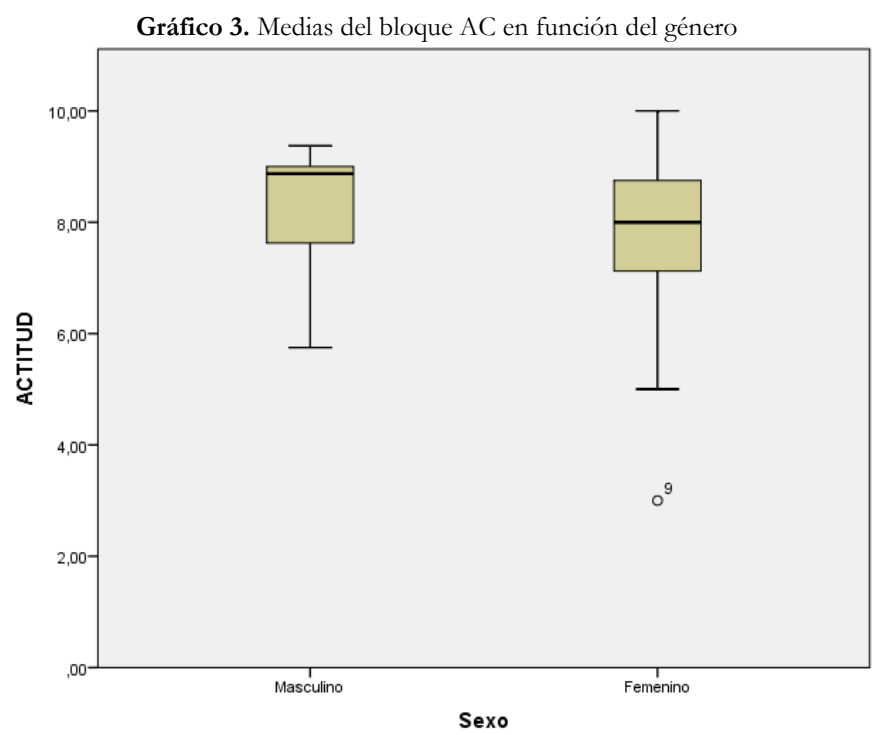

Fuente: elaboración propia. 
Por lo tanto, podemos verificar la existencia de diferencias en función del género, pudiéndose considerar, una vez aplicada la prueba U Mann-Whitney, solamente como significativas las referentes al bloque de $\mathrm{CN}(\mathrm{p}=.016)$ y al de MJ ( $\mathrm{p}=.047)$ (tabla 7).

Tabla 7. Prueba U Mann-Witney en función del género

\begin{tabular}{|c|c|c|c|c|c|c|c|}
\hline \multicolumn{8}{|c|}{ Dimensiones } \\
\hline & \multicolumn{7}{|l|}{ Género } \\
\hline & \multirow[t]{2}{*}{$\mathbf{U}$} & \multirow[t]{2}{*}{$\mathbf{Z}$} & \multirow[t]{2}{*}{ p. } & \multicolumn{2}{|l|}{ Hombre } & \multicolumn{2}{|l|}{ Mujer } \\
\hline & & & & $\bar{x}$ & $\mathrm{SD}$ & $\bar{x}$ & SD \\
\hline$C N$ & 129,500 & $-2,418$ & ,016 & 6,44 & 2,29 & 4,78 & 1,76 \\
\hline MJ & 153,000 & $-1,986$ &, 047 & 6,45 & 1,70 & 5,40 & 1,40 \\
\hline$A C$ & 205,000 & $-1,031$ & ,303 & 8,27 & 1,13 & 5,54 & 1,42 \\
\hline
\end{tabular}

\subsubsection{VARIABLE DIFERENCIADORA EDAD}

En el primer bloque (CN_01) relacionado con los conocimientos sobre conceptos TIC, las puntuaciones son mejores en los estudiantes de más de 25 años, quienes son los de más edad de la muestra estudiada (gráfico 4).

Gráfico 4. Medias del bloque CN en función de la edad

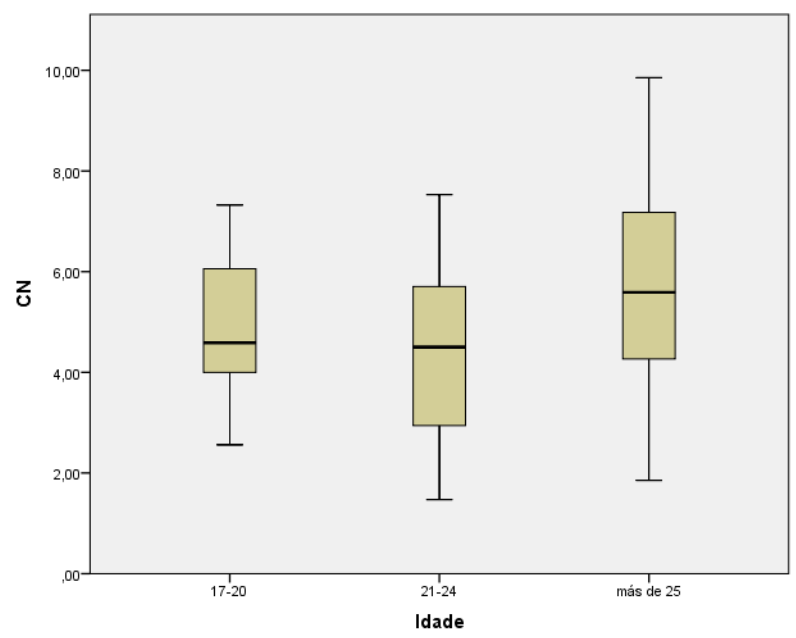

Fuente: elaboración propia. 
En cuanto al manejo de dispositivos (MJ_01), herramientas (MJ_02) y servicios (MJ_03) son igualmente los alumnos más mayores los que se autoevalúan ligeramente más alto que el resto de los subgrupos analizados (gráfico 5).

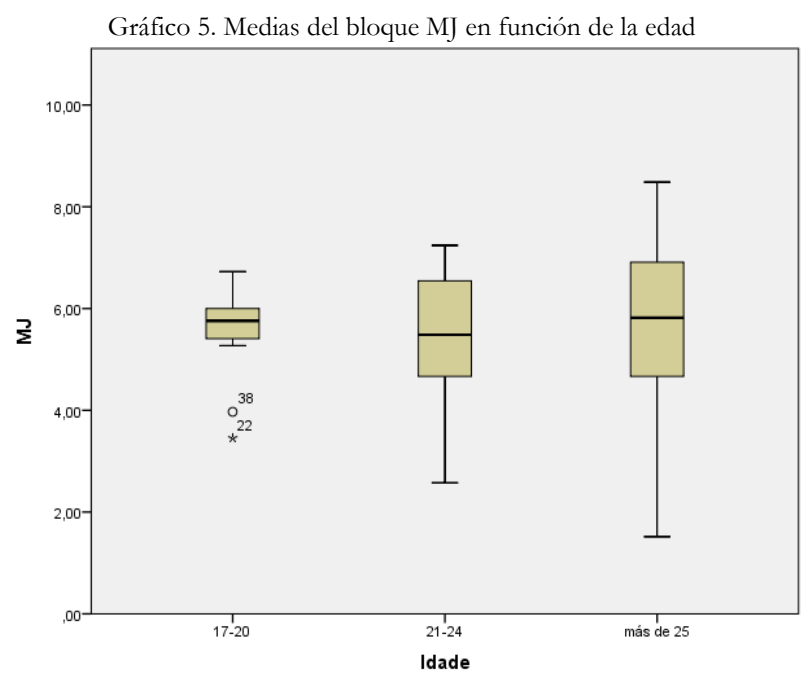

Fuente: elaboración propia.

Respecto a la actitud (AC), son los más mayores (+ 25 años), seguidos de los más jóvenes (17-20 años), los más positivos. Y los de 21-24 años son los que tienen una menor actitud hacia las TIC (gráfico 6).

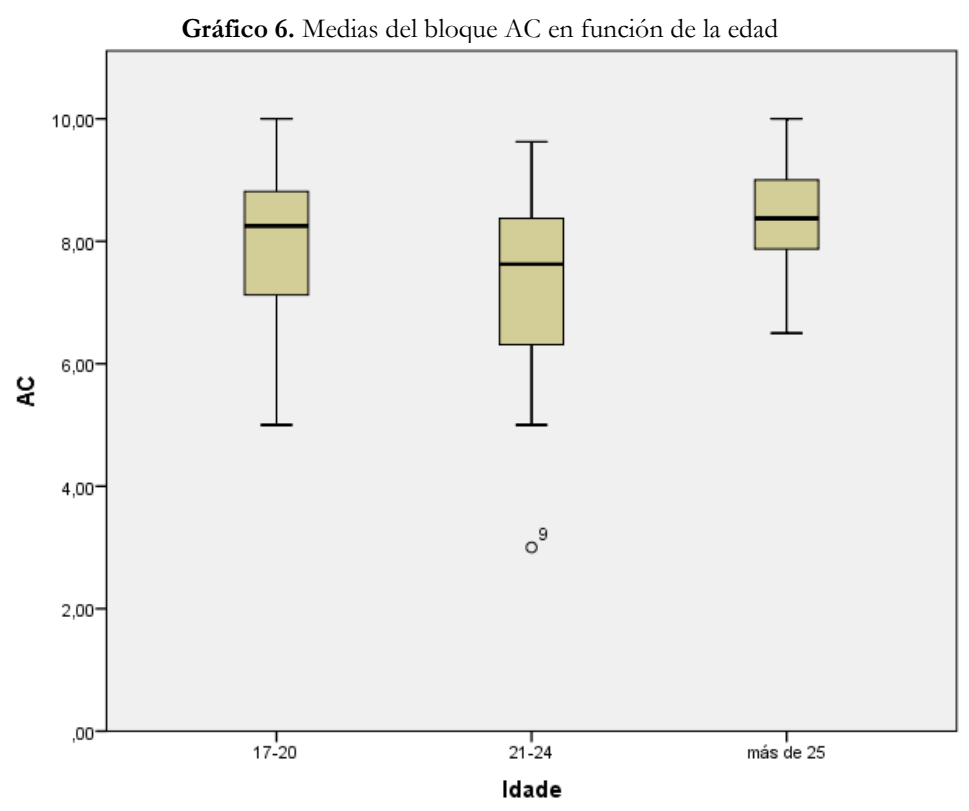

Fuente: elaboración propia. 
Al realizar la prueba de Kruskal-Wallis, comprobamos que solo se pueden catalogar como significativas $(<, 05)$ las diferencias existentes en la dimensión AC, no pudiéndose identificar como tales ni las de $\mathrm{CN}$ ni las de $\mathrm{MJ}$ (gráfico 8).

Tabla 8. Prueba Kruskal-Wallis en función de la edad

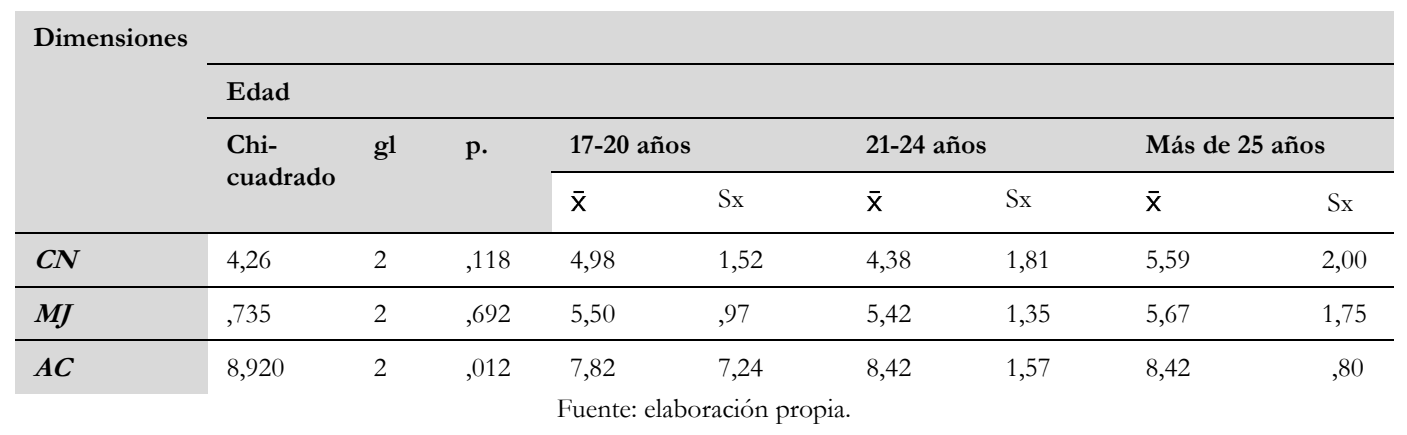

\section{CONCLUSIONES Y DISCUSIÓN DE RESULTADOS}

En este trabajo se ha identificado el nivel de competencia digital autoevaluado por futuros profesionales de la educación, centrándose en las dimensiones de conocimiento, manejo y actitud hacia las TIC y en las variables de género y edad.

Las personas que han formado parte de la muestra pueden caracterizarse como «usuarios multidispositivo», ya que la mayoría de los sujetos poseen ordenadores, Smartphone, cámara de fotos digital, Tablet y GPS.

Si tenemos en cuenta las dimensiones objeto de estudio, los futuros profesionales de la educación se suspenden en el conocimiento de términos relacionados con las TIC y su conocimiento de dispositivos tecnológicos es alto. Su valoración sobre el manejo de dispositivos es notable y reconocen su mayor competencia para la utilización de ordenadores y Tablets y su menor capacidad para el empleo de aparatos lectores de libros digitales o e-Book. Solo se suspenden en el manejo de wearables. Su autoevaluación sobre la utilización de herramientas es alta, considerándose capacitados, sobre todo, para el uso de redes sociales, herramientas de comunicación (como WhatsApp) y herramientas de edición de textos. Sus peores valoraciones se sitúan en el manejo de herramientas para la edición de imagen y sonido. Si tenemos en cuenta el uso de servicios, las autoevaluaciones son muy dispersas; en general utilizan Internet para publicar y compartir fotos y vídeos, y no tienen ni blog, ni sitio web, ni emplean el almacenamiento en la nube. Su actitud hacia las TIC es muy positiva, considerando la tecnología como necesaria y útil para su futuro profesional, además de ayudarles a economizar esfuerzo tanto en el trabajo académico como en el profesional. Respecto a esta última dimensión, otorgan su peor valoración a la afirmación: «las TIC son un medio frío y distante». Resultados similares podemos encontrar en otras investigaciones sobre el tema (Ausín y Delgado, 2015; Centeno y Cubo, 2013; Hernández, López y Sánchez, 2014).

Si tenemos en cuenta la influencia de las variables de género y de edad en las tres dimensiones, sí se encuentran diferencias significativas. 
La influencia de la variable de género en la adquisición de la competencia digital ha sido objeto de estudio de distintas investigaciones. En unas se señalan la existencia de diferencias significativas (Francis y Katz, 1996; Cabero, Llorente y Puentes, 2008); en otras, no (Centeno y Cubo, 2013; Roblizo y Cózar, 2015; Torres y Arras, 2011). En el imaginario colectivo subyace la idea de que son los hombres los que se autoperciben con un mejor nivel de competencia digital; sin embargo, estudios recientes concluyen lo contrario al indicar diferencias a favor de las mujeres (Arras, Torres y García-Valcárcel, 2011; Cózar y Roblizo, 2014; Marín y Cabero, 2010). Con nuestra investigación hemos verificado que los hombres se valoran por encima de las mujeres en conocimientos, manejo y actitud, siendo solo significativas las diferencias en las dos primeras dimensiones.

Del mismo modo, ha sido objeto de estudio la variable de edad. En algunas ocasiones, con resultados que indican diferencias a favor de los más jóvenes (García, Ramírez y Rodríguez, 2014; Martos, Pérez y Bernal, 2016), e incluso señalan el aumento de problemas de uso de las TIC con la edad (De Oleo y Rodríguez, 2010). En otros casos, no se muestran estas diferencias (Romero y Minelli, 2011). En nuestro estudio, sí se han encontrado diferencias significativas, solo en la dimensión de actitud, a favor de los más mayores de la muestra (más de 25 años), seguidos de los más jóvenes (17-20 años). También se han encontrado diferencias en el conocimiento y el manejo (a favor de los más mayores), no pudiendo ser consideradas como significativas.

\section{BibLIOGRAFÍA}

Alonso, L. (2013). Del Homo Videns al Homo Iconicus en la Sociedad del Conocimiento. Revista Filosofía, 24, 127-139. Recuperado de http://erevistas.saber.ula.ve/index.php/filosofia/article/view/4873

Arnal, J., Del Rincón, D. y Latorre, A. (1992). Investigación educativa. Fundamentos y metodología. Barcelona: Labor.

Arras, A. M. G., Torres, C. A. y García-Valcárcel, A. (2011). Competencias en Tecnologías de la Información y la Comunicación (TIC) de los estudiantes universitarios. Revista Latina de Comunicación Social, 66, 130-152. doi: https://doi.org/10.4185/RLCS-66-2011-927-130-152

Ausín, V. y Delgado, V. (2015). Aprendizaje percibido y actitud hacia las TIC desde la perspectiva de los PLE. Opción, 31(5), 91-110.

Berends, M. (2006). Survey Methods in Educational Research. En J. L. Green, G. Camilla y P. B. Elmore (Eds.), Handbook of Complementary Methods in Education Research (pp. 623-641). Washington: AERA.

Cabero, J. (2014). Formación del profesorado universitario en TIC. Aplicación del método Delphi para la selección de los contenidos formativos. Educación XXI, 17(1), 111-132. doi: https://doi.org/10.5944/educxx1.17.1.10707

Cabero, J., Llorente, M. C. y Puentes, A. (2008). Alfabetización Digital: Un estudio en la Pontificia Universidad Catolica Madre y Maestra. Sevilla: Fortic.

Cabezas, M., Casillas, S. y Pinto, A. M. (2014). Percepción de los alumnos de Educación Primaria de la universidad de Salamanca sobre su competencia digital. EDUTEC. Revista Electrónica de Tecnología Educativa, 48, 1-14. Recuperado de http://www.edutec.es/revista/index.php/edutece/article/view/156

Castells, M. (1997). La era de la información: economia, sociedad y cultura. La sociedad red. Madrid: Alianza Editorial.

Castells, M. (2006). La Sociedad Red: Una visión global. Madrid: Alianza. 
Centeno, G. y Cubo, S. (2013). Evaluación de la competencia digital y las actitudes hacia las TIC del alumnado universitario. Revista de Investigación Educativa, 31(2), 517-536. doi: http://dx.doi.org/10.6018/rie.31.2.169271

Cózar, R. y Roblizo, M.J. (2014). La competencia digital en la formación de los futuros maestros: percepciones de los alumnos de los Grados de Maestro de la Facultad de Educación de Albacete. RELATEC, Revista Latinoamericana de Tecnología Educativa, 13(2), 119-133. Recuperado de http://relatec.unex.es/article/view/1397

De Oleo, C. y Rodríguez, L. (2010). La usabilidad y la edad. Sociedady Utopía, Revista de Ciencias Sociales, 36, 235-246.

Dumont, H., Istance, D. y Benavides, F. (2010). The nature of Learning. Using Research to inspire practice. OECD Publications.

Edelhard, C. (2015). Educating Teachers for the New Millennium? Teacher training, ICT and digital competence. Nordic Journal of Digital Literacy, 10, 138-154.

Fraenkel, J., Wallen, N. y Hyun, H. H. (2012). How to design and evaluate research in education (8a ed.). Boston: McGraw Hill.

Fernández, J. M., Román, P. y El Homrani, M. (2017). TIC y discapacidad. Conocimiento del profesorado de educación primaria en Andalucía. Aula Abierta, 46, 65-72. doi: https://doi.org/10.17811/rifie.46.2017

Fernández, J. M. y Torres, J. A. (2015). Actitudes docentes y buenas prácticas con TIC del profesorado de Educación Permanente de Adultos en Andalucía. Revista Complutense de Educación, 26, 33-49. doi: http://dx.doi.org/10.5209/rev RCED.2015.v26.43812

Ferrari, A. (2012). Digital Competence in Practice: An Analysis of Frameworks. Luxembourg: Pulications Office of the European Union.

Francis, L. y Katz, Y. (1996). The gender stereotyping of computer use among female undergraduate students in Israel and the relationship with computer-related attitudes. Journal of Educational Media, 22(2), 79-86.

García, R., Ramírez, A. y Rodríguez, M. M. (2014). Educación en alfabetización mediática para una nueva ciudadanía prosumidora. Comunicar, 22(43), 15-23. doi: http://dx.doi.org/10.3916/C43$\underline{2014-01}$

González, M. P., Galvis, E. A. y Sánchez, J. M. (2015). Identificación de factores que afectan el desarrollo de la inclusión digital. Revista Virtual Universidad Católica del Norte, 44, 175-191. Recuperado de http://revistavirtual.ucn.edu.co/index.php/RevistaUCN/article/view/623/1158

Gillen, J. (2014). Digital literacies. New York \& London: Routledge.

Gisbert, M., Espuny, C. y González, J. (2011). Cómo trabajar la competencia digital con estudiantes universitarios. En R. Roig y C. Lavene (eds.), La práctica educativa en la sociedad de la información (pp. 57-174). Alicante: Marfil.

Hernández, M. A., López, P. y Sánchez, S. (2014). La comunicación en la familia a través de las TIC. Percepción de los adolescentes. Pulso, 37, 35-58.

Kampylis, P., Punie Y. y Devine, J. (2015). Promoción de un aprendizaje eficaz en la era digital. Un marco Europeo para Organizaciones Educativas digitalmente competentes. Unión Europea. doi: http://dx.doi.org/10.2791/54070

Kerlinger, F. y Lee, H. (2002). Investigación del Comportamiento. Métodos de Investigación en Ciencias Sociales. México: McGrawHill. 
Maderick, J. A., Shaoan, Z., Hartley, K. y Marchand, G. (2015). Preservice Teachers and SelfAssessing Digital Competence. Journal of Educational Computing Research, 0(0), 1-26. doi: https://doi.org/10.1177/0735633115620432

Marín, V. y Cabero, J. (2010). Del conocimiento del estudiante universitario sobre las herramientas 2.0. Anales de la Universidad Metropolitana, 10(2), 51-74.

Martos, E., Pérez, P. y Bernal, J. (2016). Relación entre la edad del profesorado de música andaluz y el desarrollo de la Escuela TIC 2.0. Revista Complutense de Educación, 27(2), 757-777. doi: http://dx.doi.org/10.5209/rev_RCED.2016.v27.n2.48264

Mork, F. y Krumsvik, R. J. (2016). Prepared to teach ESL with ICT? A study of digital competence in Norwegian teacher education. Computers \& Education, 97, 1-20. doi: http://dx.doi.org/10.1016/i.compedu.2016.02.014

Nasah, A., DaCosta, B., Kinsell, C. y Seok, S. (2010). The digital literacy debate: An investigation of digital propensity and information and communication technology. Educational Technology Research and Development, 58(5), 531-555.

Rambousek, V., Stipek, J. y Vanková, P. (2016). Contents of digital literacy from the perspective of teachers and pupils. Procedia, Social and Behavioral Sciences, 217, 354-362. doi: https://doi.org/10.1016/j.sbspro.2016.02.101

Roblizo, M. J. y Cózar, R. (2015). Usos y competencias en TIC en los futuros maestros de Educación Infantil y Primaria: Hacia una alfabetización tecnológica real para docentes. Pixel-Bit, Revista de Medios y Educación, 47, 23-39. doi: http://dx.doi.org/10.12795/pixelbit.2015.147.02

Roldán, L. A. y Zabaleta, V. (2016). Lectura y escritura. Autopercepción del desempeño en estudiantes universitarios. Revista Iberoamericana de Diagnóstico y Evaluación e Avalição Psicológica, RIDEP, 2(42), 27-38. doi: http://dx.doi.org/10.21865/RIDEP42 27

Romero, M. y Minelli, J. (2011). La generación net se tambalea: percepción del dominio de las TIC de estudiantes de magisterio. Teoría de la Educación, Educación y Cultura en la Sociedad de la Información, 12(3), 280-298.

Ricoy, M. C., Feliz, T. y Sevillano, M. L. (2010). Competencias para la utilización de las herramientas digitales en la sociedad de la información. Educación XXI, 13(1), 199-219.

Torres, C. A. y Arras, A. M. (2011). Percepción de estudiantes de educación superior sobre sus competencias en las TIC en la universidad Autónoma de Chihuahua y Veracruzana. Apertura, revista de innovación educativa, 3(2). Recuperado de http://www.redalyc.org/pdf/688/68822737009.pdf

Unión Europea (2007). Competencias clave para el aprendizaje permanente. Un marco de referencia europeo. Luxemburgo: Oficina de Publicaciones Oficiales de las Comunidades Europeas. Recuperado de http://www.mecd.gob.es/dctm/ministerio/educacion/mecu/movilidadeuropa/competenciasclave.pdf?documentId $=0901 \mathrm{e} 72 \mathrm{~b} 80685 \mathrm{fb} 1$ 